\title{
The Effect of a Commercial Game on Children's Block Design Scores on the WISC-R IQ Test*
}

\author{
JEAN DIRKS \\ Institute for the Study of Mental Retardation \\ and Related Disabilities, University of Michigan \\ 130 South First Street, Ann Arbor, MI. 48104
}

\begin{abstract}
Two experiments investigated the extent to which 10-year old children's scores on the WISC-R Block Design subtest were affected by prior experience with a specific commercial game that involved blocks and matching patterns. Experiment 1 found that 12 10-year old children who happened to have experience with the particular commercial game scored approximately three scaled score points higher on the WISC-R Block Design subtest than 24 matched children without game experience. In Experiment 2, 24 children who did not have prior experience with this particular commercial game were randomly assigned either to a Game condition (involving two 15-minute sessions with the game) or to a No-Game condition (which involved no further game experience). Children in the Game condition subsequently increased their WISC-R Block Design scores more than children in the No-Game condition. Taken together, the experiments indicate that relatively brief interactions with a commercial game can cause a significant improvement in children's performance on an IQ subtest.
\end{abstract}

In much of the previous research on individual differences in IQ scores, attention has been focused on possible connections or lack of connections between IQ scores on the one hand, and various large-scale "molar" variables such as social class or race. The current article focuses on the relation between IQ and environment, but examines a fairly "molecular" aspect of the environment. The question examined here is whether children's performance on a standardized test of intelligence can be elevated by prior experience with a commercial game.

*The author was supported during different parts of this research by grant \#5 R01 MH32326 from the National Institute of Mental Health and by a National Institutes of Health traineeship \#T32 HD07109. Romayne Jackson, Joanne Quarfoth, and Deborah Simmons assisted in gathering data or providing game experience to subjects. Robert Sternberg and Fred Royer helped critique an earlier version of this paper. Address for reprints: Dr. Jean Dirks, Southgate Regional Center for Developmental Disabilities, 16700 Pennsylvania Road, Southgate, MI 48195. 
At the moment, there is some indication that infants' and children's cognitive and perceptual skills may be more advanced when the subjects have the opportunity to interact with various games and objects. For example, infants and young children who have access to appropriate play materials do better than less advantaged controls on subsequent intelligence tests (Bradley \& Caldwell, 1976a, $1976 \mathrm{~b}$ ), and children who have the opportunity to observe or manipulate masses of clay being shaped do better on conservation of mass tasks than children who lack this experience (Price-Williams, Gordon, \& Ramirez, 1969).

Similarly, children who come from a culture that provides experience in constructing and playing a seriated array of bamboo pipes are subsequently more successful in other seriation tasks than children from cultures without commensurate exposure to the seriated pipes (Stevenson, Parker, Wilkinson, Bonnevaux, \& Gonzalez, 1978). Finally, interaction with mobiles and toys in general is often a built-in component to environmental intervention programs which succeed in raising infants' or preschoolers' IQ scores (Karnes, Teska, Hodgins, \& Badger, 1970; Scarr-Salapatek \& Williams, 1973).

All of these findings suggest that young children can improve their perceptual and cognitive skills—and in some cases their IQ scores in particular-through exposure to and interaction with objects. Because these findings are based on studies involving fairly long-term exposure to the objects in question, however, little is known about the effect of short-term exposures such as might occur when children are introduced to and play a commercial game for a brief period in their life. Since many commercial games claim to provide "educational" and training experiences, it would be particularly interesting to ascertain just how commercial games affect scores on standardized tests.

In order to obtain quantitative information on this issue, the current study examined whether commercial game playing could affect 10-year old children's scores on a standardized test of intelligence, the Wechsler Intelligence Scale for Children-Revised (WISC-R). The study focusses on a particular commercial game called "Trac 4" that bears a very close resemblance to one of the subtest tasks, the Block Design subtest, which appears on the WISC-R test itself. This game is one of a number of commercial games which mimic to lesser or greater extents the tasks that are involved on various IQ tests.

The current game was selected for study in part because the IQ subtest which it resembles (Block Design) is often accorded particular emphasis because it correlates highly with Full-Scale WISC-R IQ (Wechsler, 1974). In addition, the Block Design subtest is often used when various "short-form" or "quick" estimates of children's mental abilities are obtained. For example, the WISC-R combination of Block Design and Vocabulary subtests is traditionally considered the best shortform estimate of WISC-R IQ (Sattler, 1974) and it has been used by school psychologists or by researchers who wish to obtain a quick estimate of IQ. Because of this, it is especially important to determine what Block Design scores signify, and whether IQ estimates derived from Block Design short forms are unduly influenced by chance environmental opportunities. 


\section{DESCRIPTION OF COMMERCIAL GAME}

The commercial game involved here, called Trac 4, is put out by Lakeside Toy Company and retails for approximately $\$ 12$ to $\$ 15$. On both the game and the Block Design subtest, children attempt to arrange three-dimensional cubes so as to duplicate the geometric pattern in a pictured square model. Each cube has two red sides, two white sides, and two sides which are divided along the diagonal into a red half and a white half. In both the game and the Block Design subtest, the successful participant must produce a totally accurate copy in a speedy manner.

In addition to these basic similarities, there are some differences between the game and the Block Design subtest. For example, in the commercial game, multiple players race against each other and the pattern to be matched is only briefly and intermittently visible since it revolves in and out of sight on a large, noisy, revolving timer. In the Block Design subtest by contrast only one player (the $S$ being tested) is present, the pattern to be matched is stationary and continuously in view, and the timer (a stopwatch) is small and relatively inconspicuous. In general, all of these differences make the element of time more salient in the game situation than in the Block Design subtest.

Some other differences between the game and the Block Design subtest are illustrated in Figure 1. Specifically, the game has more blocks per design than the Block Design subtest ( 16 per game pattern vs. 4-9 per Block Design pattern). Accordingly, the game provides more extensive practice in matching blocks with diagonals than the Block Design subtest, since each game player will have the opportunity to match 68 diagonals (if he/she completes one round at all difficulty levels), while each Block Design subject aged eight years or older will have the opportunity to match only 35 diagonals (if he/she completes all difficulty level Block Design patterns). A final difference between the game and the Block Design subtest is that the game involves a superimposed grid over the pictured design, which makes it easier for game players to segment the array into rows and columns of blocks. These differences will be commented on in more detail in Experiment 2.

\section{TRAC 4}

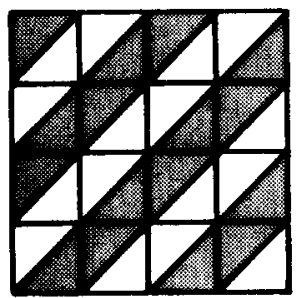

WISC- R

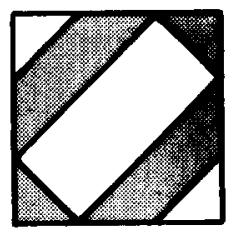

FIG. 1. Sample patterns to be matched in Trac 4 game and WISC-R Block Design subtest. 


\section{EXPERIMENT 1}

Experiment 1 investigated whether naturally occurring variations in children's Block Design scores were related to differences in exposure to the commercial game described above.

\section{Subjects}

The subjects were fourth graders in a small middle-class to lower-middle-class town in Michigan. Fourth graders were selected for study because the game was judged to be particularly suitable for children in grades $2-7$.

When the fourth graders in two schools were questioned with a copy of the game in view as to their prior experience with that game, 12 non-retarded children (11 boys and 1 girl) reported that they had played the game on two or more occasions prior to being administered a WISC-R test that year. These $S$ s were designated "Game-Experienced." There were 133 children (78 boys, 55 girls) who had not played the game and who were designated "Game-Inexperienced." The relatively high proportion of boys among the Game-Experienced $S$ s confirms Rheingold and Cook's (1975) finding that parents tend to buy more "spatialtemporal" and more "educational" games for their sons than for their daughters.

As part of another study on the usage of short-form IQ tests and the identification of gifted children (Dirks, Wessels, Quarfoth, \& Quenon, 1980; Dirks \& Quarfoth, 1981), all 12 Game-Experienced $S s$ and 78 Game-Inexperienced $S s$ had been administered the Wechsler Intelligence Scale for Children-Revised (WISC-R). For all but three subjects, the WISC-R tester had been unaware at the time of testing whether the child being tested was game-experienced or gameinexperienced. WISC-R testers were women who had previous university training and experience in the administration of WISC-R tests.

For purposes of the current study, the 12 Game-Experienced $S \mathrm{~s}$ were matched to 24 Game-Inexperienced controls. The control $S s$ were selected by matching each Game-Experienced $S$ to the two same-sex Game-Inexperienced $S$ s who had the closest WISC-R Verbal IQ to their Game-Experienced match. If several possible matches were equally close in Verbal IQ to a particular Game-Experienced $S$, the selection was made that would minimize the difference in Verbal IQ between Game-Experienced $S s$ as a group and Game-Inexperienced $S s$ as a group. Matching was done on the basis of sex and Verbal IQ to insure that GameExperienced and Game-Inexperienced $S$ s would be roughly comparable in background and general learning ability. In the matching, any given GameInexperienced $S$ was used for at most one match, so that there were 24 different control Ss.

The mean age of the $S \mathrm{~s}$ was 10.1 years for the 12 Game-Experienced $S \mathrm{~s}$ and 10.0 years for the 24 Game-Inexperienced $S s$. Each Game-Experienced $S$ and his/her matched controls differed by no more than 5 Verbal IQ points. 


\section{RESULTS}

Table 1 summarizes the WISC-R data. The five Performance subtests require the $S$ to arrange blocks so as to match a geometric pattern (Block Design), pick out missing details in pictures (Picture Completion), arrange pictures in logical sequence (Picture Arrangement), assemble puzzles (Object Assembly), and learn paired associations between numbers and visual patterns (Coding). In these subtests, the $S$ does not respond verbally but points to or manipulates tangible material. The Performance IQ score is based on the summed scores across the five Performance subtests.

The Verbal IQ score is based on five subtests which require the $S$ to respond verbally to questions concerning factual information, vocabulary, arithmetic, verbal similarities, and consequences and causes of various environmental situations. The Full Scale IQ score is based on the composite scores across all Verbal and Performance subtests.

To begin with, a two-way analysis of variance with repeated measures (least squares solution for unequal $\boldsymbol{n}$ design) was carried out on the scaled scores from the five Performance subtests. The variables were condition (Game-Experience vs. No Game-Experience) and Subtest (the five Performance subtests). There was a significant effect of Subtest $(F(4,136=8.66, p<.001)$ and no overall effect of Game Experience $(F(1,34)=2.24, p>.10)$.

However, the most important finding was a significant interaction between Subtest and Game Experience $(F(4,136)=3.34, p<.05)$. In particular, when $t$-tests were applied to data from individual subtests, Game-Experienced Ss scored significantly higher than Game-Inexperienced controls on the Block Design subtests $(t(34)=3.39, p<.001)$. Table 1 indicates that Game-Experienced $S$ s scored approximately three scaled score points higher on the Block Design subtest than Game-Inexperienced Ss. However, there were no significant differences on $t$

TABLE 1

WISC-R Scores Obtained in Experiment 1

\begin{tabular}{lcc}
\hline Test Measure & $\begin{array}{c}\text { Game-Experienced } \\
\text { Subjects }\end{array}$ & $\begin{array}{c}\text { Game-Inexperienced } \\
\text { Subjects }\end{array}$ \\
\hline $\begin{array}{l}\text { Mean Scaled Score on } \\
\text { Performance subtests }\end{array}$ & & \\
$\quad$ Block Design & 15.1 & 11.9 \\
Picture Compl. & 12.8 & 12.0 \\
Picture Arrgt. & 12.3 & 12.3 \\
Object Assembly & 12.7 & 13.0 \\
Coding & 10.5 & 10.2 \\
Verbal IQ & 115.1 & 115.3 \\
Performance IQ & 118.8 & 113.0 \\
Full Scale IQ & 118.8 & 116.0 \\
\hline
\end{tabular}


tests between Game-Experienced Ss and Game-Inexperienced controls on any of the other four Performance subtests.

In order to determine what effect the commercial game had on the Ss' overall Performance IQ score, a $t$-test was used to compare the Performance IQ scores of the 12 Game-Experienced $S s$ and the 24 Game-Inexperienced controls. This analysis indicated that Game-Experienced $S$ s obtained marginally higher Performance IQ scores than Game-Inexperienced controls $(t(34)=1.53, .05<p<.10)$. It is important to add that in the Wechsler (1974) scoring manual, a 3-point increase in any one Performance subtest (which is the size of the current effect of game experience) will automatically cause a 3-5 point elevation in performance IQ score in that $S$.

\section{DISCUSSION}

At the outset, two alternative explanations for the Block Design scores are possible: Either the Block Design scores caused the extensive game experience, or alternatively the game experience caused the high Block Design scores.

According to the first possibility, children who already had high Block Design scores might have been particularly motivated to spend a lot of time playing a game that gave them a chance to use and show off their particular skills with blocks or with matching. This possibility-which is that high Block Design scores caused the game experience-did seem unlikely however when the overall IQ data from the fourth grade as a whole was examined. In the fourth grade, game experience was not specific to high Block Design scores per se, since there was another subset of 24 Game-Inexperienced children who had identical Block Design scores to the Game-Experienced $S$ s.

Interestingly enough, Game-Experienced Ss' Block Design scores were 3.0 scaled score points higher than the mean of their other four Performance subtests, while this new subset of 24 Game-Inexperienced $S$ s had only a 1.8 point difference between their Block Design scaled scores and their other four Performance subtest scores. This suggests then that game-experience was not specific to high Block Design scores per se, but it was more likely to occur in the particular group of $S \mathrm{~s}$ whose Block Design scores were unusually higher than their other scores. All of this makes it unlikely that high absolute Block Design scores caused the high game experience.

The alternative explanation, which is favored here, is that game experience actually caused the high Block Design scores. It is concluded here that children who played the commercial game on several occasions developed certain psychological skills which in turn were easily applied and put to good use during the Block Design task. In other words, "transfer of training" is assumed to have occurred between the commercial game and the Block Design task. This conclusion is further buttressed by Experiment 2 . 


\section{EXPERIMENT 2}

If the preceding interpretation is correct, then it should be possible to experimentally produce an improvement in children's Block Design scores by giving a child prior experience with the commercial game in question. This was done in Experiment 2.

In Experiment 2, children were randomly assigned to receive either game experience or no game experience, and their scaled scores on the Block Design subtest were assessed before ("Pretest") and after ("Post-test") the assignment had been fulfilled. If children with game experience showed greater improvement between their pretest and post-test Block Design scores than children without game experience, then it could definitely be concluded that game experience caused the improvement in Block Design scores.

In order to provide further information on the effect of game experience, Experiment 2 also recorded data concerning three psychological skills that might conceivably explain why game experience could cause improvement on the Block Design task: (1) Overall motivation to hurry; (2) Usage of systematic copying sequence; and (3) Accurate matching of diagonals.

Motivation to Hurry. At the beginning of this article, it was noted that one of the main differences between the commercial game and the Block Design task was that game players were likely to be more aware of time due to the presence of racing competitors, a large conspicuous timer, and a visual pattern that was only briefly and intermittently visible. If extended game experience led players to become more conscious of time, and to be more motivated to hurry, then this could lead to increases in overall finger speed and visual scanning speed which in turn would result in improved Block Design scores during later testing.

In order to determine if this in fact is occurring, $S \mathrm{~s}$ in Experiment 2 were given pre-test and post-test assessments not only on the Block Design subtest, but also on another task which called for rapid manipulation of fingers and rapid visual processing. This task, which was the WISC-R Object Assembly subtest, required $S$ s to use their hands to put together pieces of a puzzle as raptdly as possible. It was hypothesized here that if game experience had produced an overall increase in speed of movements, then Game-Experienced $S$ s should show greater improvement on the Object Assembly test (as well as on the Block Design subtest) than Game-Inexperienced Ss.

Usage of Systematic Copying Sequence. Another way in which game experience might affect psychological skills might be through the influence of the superimposed grid. As noted in Figure 1 earlier in this article, the commercial game had a superimposed grid over each pattern, while the Block Design task did not.

Anecdotal observation of game players suggested that this grid in effect provided a ready-made copying strategy for players, since it effectively segmented 
the array into the correct arrangement of rows and columns. In general, game players use this grid to adopt a systematic copying strategy, since they typically begin in one corner of the grid and then simply go up and down the grid columns or back and forth across the grid rows as they match each block in order.

By contrast, anecdotal observation of Block Design players indicates that Block Design players are less likely to use this systematic copying strategy. On the more difficult Block Design patterns in particular, the young Block Design player is often at a loss as to how to segment the pictured array. Since they are without a grid, Block Design players are sometimes unaware that the array actually involves rows and columns, and their copying strategy can thus become a haphazard "skip-around" strategy where they move slowly from one place in the design to another at random. The relative difficulty of ungridded designs for performance in general has been observed not only by the author in anecdotal observations, but also by Royer $(1977,1978)$ in his study on adult performance on block design-like tasks.

Taken together, these observations suggest that one of the possible effects of game experience might be that it tends to create a systematic copying sequence. Game-experienced players may thus be more likely than game-inexperienced players to create their own imaginary grid when they come to the Block Design task. They may therefore be more likely than game-inexperienced players to adopt a systematic copying sequence on the Block Design task by copying in sequence along rows or columns of the imagined grid.

Accurate Matching of Diagonals. Although subjects in general have little difficulty in matching solid color areas, they may have trouble in matching or copying patterns that involve diagonals (Royer, 1977, 1978; Olson, 1970). This difficulty arises in the first place from an occasional tendency to copy diagonals in reverse, so that a "northeast to southwest" diagonal may be copied for example with a "northwest to southeast" line. This difficulty also arises in the second place because $S s$ sometimes think that the diagonal line is the boundary between two different blocks, rather than a line falling in the middle of a single block.

As noted earlier in this paper however, the commercial game affords particularly rich practice in matching diagonals, since each player who goes through a complete sequence at all difficulty levels will thereby have matched 68 blocks involving diagonals. If diagonal-matching improves with practice, it therefore might be expected that game-experienced $S$ s would show less tendency to copy diagonals "in reverse" than game-inexperienced Ss.

In addition, the superimposed grid which is present in the commercial game provides players with an automatic means for determining where between-block boundaries fall, and thus reduces the second cause of diagonal errors cited above. If game-experienced players tend to imitate the game by creating their own imaginary grid during the Block Design task, game-experienced players should thus re- 
alize that diagonal lines-which never coincide with grid lines-cannot form between-block boundaries.

Both of these interpretations then suggest that game-experienced players should be more accurate in copying blocks with diagonal lines than gameinexperienced players. This hypothesis is tested in Experiment 2, along with the earlier hypotheses regarding copying sequence and motivation to hurry.

\section{METHODOLOGY}

\section{Procedure}

In Experiment 2, 24 9- to 11-year-old boys were selected who lacked prior experience with the Trac 4 commercial game. Each $S$ was given a pretest on the WISC-R Block Design subtest and the WISC-R Object Assembly subtest. During the Block Design subtest, information was recorded on the overall WISC-R scaled score, the number of solid-color and diagonal-pattern blocks that were matched accurately, and the type of copying sequence adopted. Children were then randomly assigned to either a Game Experience or a No-Game condition, with the proviso that the mean Block Design and Object Assembly pretest scores of the Game Experienced group had to be within one scaled score point of the scores of the No-Game group.

The 12 children in the Game condition were then given two 15-minute sessions playing the Trac 4 game. The two sessions were approximately 7-14 days apart. All children (including those in the Game and in the No-Game conditions) were then tested afterwards on the WISC-R Block Design and WISC-R Object Assembly subtests. The mean time between pretest and post-test was 20 days for $S s$ in the Game condition, and 24 days for $S$ s in the No-Game condition.

Testing was carried out by two female testers who each had at least two years' experience in WISC-R testing. One tester was blind as to which Ss were in the Game vs. No-Game condition, while the other tester was not blind in this regard. There was no indication that the tester knowledge in this respect biased the data, since a post-hoc analysis of the WISC-R data revealed that the improvement between pretest and post-test scores was highly similar for the children tested by the different testers.

\section{Subjects}

The $S$ s were 24 boys aged $9-11$ years. The mean age was 9 years 10 months for Game $S s$, and 10 years 0 months for No-Game $S$ s. The $S$ s were members of a boy scout troop from a middle class neighborhood in a small town in Michigan. At the start of the study, the $S$ s had not had any prior experience with the Trac 4 game. 


\section{Results}

Table 2 summarizes the WISC-R data, while Figure 2 graphs the Block Design data.

The Block Design scores represented in Figure 2 were analyzed with a $2 \times 2$ (Condition $\times$ Time) analysis of variance, with repeated measures over time (PreTest vs. Post-Test). There was a significant effect of Time $(F(1,22)=42.38$, $p<.001$ ), which indicated that post-test scores were higher than pretest scores. There was no significant effect due to Condition (Game vs. No-Game) $(F(1,22)=3.02, p<.10)$, but there was a highly significant Condition $\times$ Time interaction $(F(1,22)=21.26, p<.001)$.

As indicated in Table 2 and Figure 2, the Condition $\times$ Time interaction arose because $S s$ in the Game condition increased their Block Design scores between pretest and post-test by a larger amount than $S \mathrm{~s}$ in the No-Game condition (increase of 3.4 vs. 0.6 scaled score points for Game vs. No-Game $S$ s).

TABLE 2

WISC-R Scores Obtained in Experiment 2

\begin{tabular}{llcr}
\hline & \multicolumn{3}{c}{ Subtest Scaled Scores: } \\
\cline { 2 - 5 } Subtest and Subject Group & Pre-Test & Post-Test & Change \\
\hline Block Design Subtest & & & \\
$\quad$ Game Ss & 11.8 & 15.2 & +3.4 \\
$\quad$ No-Game Ss & 10.9 & 11.5 & +0.6 \\
Object Assembly Subtest & & & \\
$\quad$ Game Ss & 11.3 & 13.8 & +2.5 \\
$\quad$ No-Game Ss & 10.8 & 12.8 & +2.0 \\
\hline
\end{tabular}

Possible Reasons for the Effect of Game Experience. Did any of the three psychological skills mentioned previously contribute to this beneficial effect of game experience? The bottom half of Table 2 indicates that apparently, game experience did not cause an overall increase in finger speed and/or visual scanning speed. That is, on another perceptual-motor task (Object Assembly) which also required rapid finger movements and rapid visual scanning, game experienced $S$ s improved about as much as $S s$ without game experience. This was confirmed statistically when a two-way ANOVA-using Time (Pretest vs. Post-Test) and Condition (Game vs. No-Game) as factors-found that there was no significant Time $\times$ Condition interaction for Object Assembly scores $(F(1,22)=0.53$, $p>.30$ ).

Turning to Table 3 however, the evidence is a bit more positive concerning the remaining two psychological skills mentioned earlier.

The top third of Table 3 presents the percent of $3 \times 3$ Block Design arrays in which $S$ s began the task by attempting to copy in sequence an entire imaginary row or column from the pictured design. Ss who played the game were more likely 


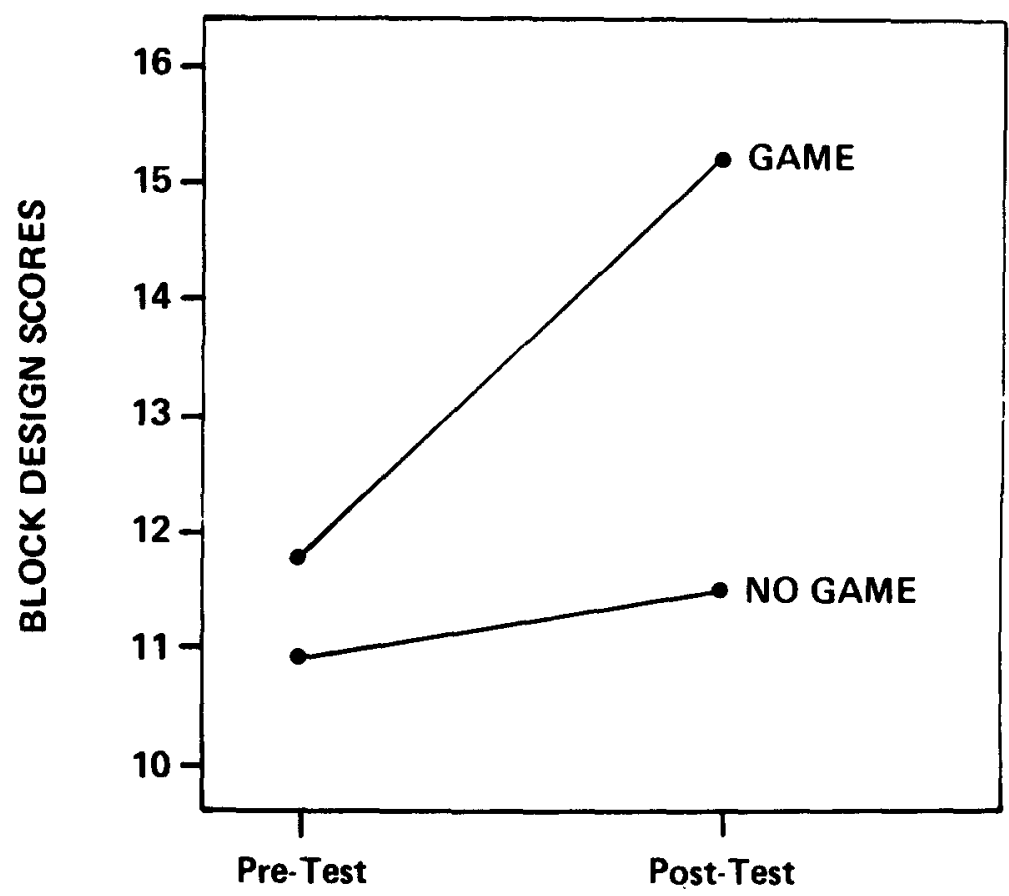

\section{TIME OF TEST}

FIG. 2. Graph of Block Design scores obtained in Experiment 2 .

TABLE 3

Change in Specific Aspects of Block Design Performance in Experiment 2

\begin{tabular}{lccr}
\hline $\begin{array}{l}\text { Aspect of Block Design } \\
\text { Performance being studied }\end{array}$ & $\begin{array}{c}\text { Data } \\
\text { on Pre-Test }\end{array}$ & $\begin{array}{c}\text { Data } \\
\text { on Post-Test }\end{array}$ & $\begin{array}{c}\text { Change over } \\
\text { time }\end{array}$ \\
\hline $\begin{array}{l}\text { \% of } 3 \times 3 \text { arrays which Ss began } \\
\text { copying with an ordered sequence } \\
\quad \text { Game Ss }\end{array}$ & & & \\
$\quad \begin{array}{l}\text { No-Game Ss } \\
\text { \% of squares with diagonal pat- }\end{array}$ & $40 \%$ & $79 \%$ & $+39 \%$ \\
terns that were correctly copied & & $48 \%$ & $+3 \%$ \\
$\quad$ Game Ss & $70 \%$ & $85 \%$ & $+15 \%$ \\
$\quad$ No-Game Ss & $64 \%$ & $64 \%$ & $0 \%$ \\
$\begin{array}{l}\text { \% of one-color squares that were } \\
\text { correctly copied }\end{array}$ & & & \\
$\quad$ Game Ss & $88 \%$ & $92 \%$ & $+4 \%$ \\
$\quad$ No-Game Ss & $92 \%$ & $92 \%$ & $0 \%$ \\
\hline
\end{tabular}

${ }^{a}$ Data on copying sequence was based only on the 8 Game and 7 no-Game Ss who attempted $3 \times 3$ arrays during both the Pre- and Post-Test. 
to adopt this sytematic copying sequence on the Block Design Post-test than Ss who had not played the game. A Mann-Whitney test (Siegel, 1956) of the change scores in the top third of Table 3 indicated that $S$ s with game experience tended to improve more in this regard between Pre- and Post-Test than $S s$ without game experience $\left(\mathrm{U}=14.5, \mathrm{n}_{1}=7, \mathrm{n}_{2}=8, p<.07\right)$.

A similar trend appears in the bottom half of Table 3. Game experience produced a modest improvement in the ability to copy diagonal blocks accurately, but as expected it left copying of solid-colored blocks (which was already relatively accurate) unchanged. A Mann-Whitney test of the change scores in the middle third of Table 3 indicated that $S$ s with game-experience showed significantly more improvement between Pre- and Post-Test in copying diagonals than Ss without game experience $\left(\mathrm{U}=30.5, \mathrm{n}_{1}=12, \mathrm{n}_{2}=12, p<.02\right)$.

\section{DISCUSSION}

In both Experiments 1 and 2, children who had prior experience with the Trac 4 commercial game scored approximately three scaled score points higher on the WISC-R Block Design subtest than comparable children without game experience.

How does this difference affect WISC-R IQ scores? The answer to that question depends in large part on the number of WISC-R subtests which are administered. If the Block Design subtest were given in conjunction with the remaining nine WISC-R subtests, then a 3-point difference on any one subtest would automatically translate into only a 1-2 point difference in Full Scale IQ, according to the Wechsler (1974) scoring manual. If only the five Performance subtests were administered, then a 3-point difference on one subtest would cause a 3-5 point increase in Performance IQ.

In the extreme case, if the Block Design subtest was administered with just one or two other WISC-R subtests, as part of a short-form battery designed to screen school children for retarded or for gifted programs, then a 3-point difference on one subtest alone would cause a difference of approximately 8-10 IQ points in the short-form IQ. This figure is based on Sattler's (1974, pp. 165-167) procedure for calculating short-form IQ scores.

One obvious practical implication of this fact is that school psychologists would do well to avoid short-forms with Block Design in populations where Trac 4 is known to be used. In such a population, short forms with Block Design will grossly overestimate a game-experienced child's IQ score, and thus provides an inaccurate estimate of what the child's "real" IQ (based on all WISC-R subtests) might be. This conclusion was also reported in Dirks, Wessels, Quarfoth, and Quenon's (1980) article on the effect of short-form IQ tests. In that article, which analyzed the same fourth grade population from which the Ss in Experiment 1 of the current article were drawn, short forms involving Block Design provided only a mediocre (and not a good) indication of overall Full Scale IQ. 
From a larger perspective, the current article also has application to the theoretical controversy concerning the relation of intelligence test scores to environmental factors. In the current research, a specific environmental factor-experience with a particular game-had a very narrowly defined effect on one portion of an intelligence test. The narrowness of the effect can be seen by the fact that in Experiment 1 , game-experience only affected scores on the one WISC-R performance subtest that was most similar in task and stimulus materials to the environmental experience. Similarly, in Experiment 2, game experience did not affect another perceptual-motor task (Object Assembly) that also required rapid hand movement and rapid visual processing. Instead, game experience in Experiment 2 appeared to best affect that small subset of psychological skills (systematic copying sequence, and accurate matching of diagonals) that was highly specific to the particular game and which would not necessarily be shared by games in general.

The narrowness of the effect of game experience is reminiscent of the specificity of effect in cross-cultural research where school-age children showed transfer of training between experience with clay and a highly similar conservation of mass task (Price-Williams, Gordon, \& Ramirez, 1969), or where apparent transfer of training occurred between experience with seriated playing pipes and performance on a seriation task (Stevenson, Parker, Wilkinson, Bonnevaux, \& Gonzalez, 1978). This narrowness of effect may be due in part to the advanced age of the subjects. Once a subject has reached school age that is, his/her thought patterns may be so well developed and fixed that small amounts of environmental training may only be able to produce small-scale changes in closely related component skills.

In closing, it may be useful to comment on whether the current study indicates that the WISC-R is "culturally biased." Jensen $(1974 ; 1980)$ and Miehle (1979) suggest that intelligence tests are not culturally biased, since there is minimal or no Group $\times$ Item interaction, or minimal indication that specific test items which are relatively easy for one group of subjects are relatively hard-compared to other test items-for a different group of subjects. In Jensen's and Miehle's, and others' research, group membership has traditionally been defined by large-scale subject attributes such as race or social class.

The current study differs in one respect from this past research since it finds clear evidence that particular environmental experiences can cause a shift in the rank ordering of difficulty of test items. In other words, subjects who have one type of background (Game Inexperience) find Block Design tasks about as easy as other WISC-R nonverbal tasks. However, subjects who have a different type of background (Game Experience) find Block Design tasks much easier than other WISC-R nonverbal tasks.

It is important to add however that the current criteria for defining subject differences (Game experience) is much narrower than the previous criteria traditionally used (race or social class). Thus, the current study suggests that the WISC-R is culturally biased against the "culture" of people who lack experience 
with this particular commercial game, but the current study does not indicate that the WISC-R is culturally biased, for example, against economically disadvantaged people in general.

In particular, in the present study, a relatively expensive commercial game did produce elevated scores on the WISC-R. However, this does not mean that poorer families as a whole-who lack the economic means to buy expensive commercial games such as Trac 4-will do worse on the WISC-R than wealthier families. That is because there may be other economically linked factors which could have an opposite and possibly overshadowing effect on WISC-R scores. For example, if families who lacked expensive commercial games were to systematically encourage their children to play outside more, then these children might conceivably develop exceptional ability to find their way around large environments and attend to spatial dimensions and spatial landmarks. There is anecdotal evidence to suggest that environmental exploration might in turn increase these children's Block Design scores or perceptual scores to a large extent (Berry, 1966; Munroe \& Munroe, 1971), possibly making these $S$ 's scores even higher than the scores of $S$ 's with game experience.

Although this scenario is entirely hypothetical, it does point up the danger of trying to relate the current findings to large-scale molar environmental factors such as economic status. The present research does however support a more limited or small-scale environmentalist position. This research indicates that a child's brief experience with a particular commercial game can have a modest but statistically significant effect on that child ${ }^{2} s$ performance on a standardized intelligence test.

\section{REFERENCES}

Berry, J. W. Temne and Eskimo perceptual skills. International Journal of Psychology, 1966, 1, 207-229.

Bradley, R. H., \& Caldwell, B. M. Early home environment and changes in mental test performance in children from 6 to 36 months. Developmental Psychology, 1976, 12, 93-97.(a)

Bradley, R. H., \& Caldwell, B. M. The relation of infants' home environment to mental test performance at 54 months: A follow-up study. Child Development, 1976, 47, 1172-1174. (b)

Dirks, J., \& Quarfoth, J. Selecting children for gifted classes: Choosing for breadth vs. choosing for depth. Psychology in the Schools, 1981, 18, 437-449.

Dirks, J., Wessels, K., Quarfoth, J., \& Quenon, B. Can short-form WISC-R IQ tests identify children with high Full Scale IQ? Psychology in the Schools, 1980, 17, 40-46.

Jensen, A. R. How biassed are culture-loaded tests? Genetic Psychology Monographs, 1974, 40, 185-244.

Jensen, A. R. Bias in mental testing. New York: Free Press, 1980.

Karnes, M. B., Teska, J. A., Hodgins, A. S., \& Badger, E. D. Educational intervention at home by mothers of disadvantaged infants. Child Development, 1970, 41, 925-935.

Miehle, F. Cultural bias in the WISC. Intelligence, 1979, 3, 149-164.

Munroe, R. L., \& Munroe, R. H. The effect of environmental experience on spatial ability in an East African Society. The Journal of Social Psychology, 1971, 83, 15-22. 
Olson, D. R. Cognitive development: The child's acquisition of diagonality. New York: Academic Press, 1970.

Price-Williams, D. R., Gordon, W., \& Ramirez, M. III. Skill and conservation. Developmental Psychology, 1969, 1, 769.

Rheingold, H. L., \& Cook, K. V. The content of boys' and girls' rooms as an index of parents' behavior. Child Development, 1975, 46, 459-463.

Royer, F. L. Information processing in the Block Design task. Intelligence, 1977, 1, 32-50.

Royer, F. L. Intelligence and the processing of stimulus structure. Intelligence, 1978, 2, 11-40.

Sattler, J. M. Assessment of Children's Intelligence. Philadelphia, PA: W. B. Saunders Company, 1974.

Siegel, S. Nonparametric Statistics for the Behavioral Sciences. New York: McGraw-Hill Book Company, 1956.

Scarr-Salapatek, S., \& Williams, M. L. The effects of early stimulation on low-birth-weight infants. Child Development, 1973, 44, 94-101.

Stevenson, H. W., Parker, T., Wilkinson, A., Bonnevaux, B., \& Gonzalez, M. Schooling, environment, and cognitive development: A cross-cultural study. Monographs of the Society for Research in Child Development. Serial No. 175, 1978.

Wechsler, D. Manual for the Wechsler Intelligence Scale for Children-Revised. New York: The Psychological Corporation, 1974. 